\title{
Effect of the lifting of Kramer's degeneracy on excitonic linewidths in quantum well optical modulators
}

\author{
Songcheol Hong and Jasprit Singh \\ Department of Electrical Engincering and Computer Science, The University of Michigan, Ann Arbor, \\ Michigan 48109 \\ Rajeshwar Sahai and Chewlan Lastufka \\ Rockwell International, Science Center, 1049 Camino Dos Rios, Thousand Oaks, California 91360
}

(Received 30 November 1987; accepted for publication 22 June 1988)

\begin{abstract}
In symmetric quantum well structures, the hole subband states maintain the spin degeneracy (Kramer's degeneracy), but in asymmetric quantum wells and in optical modulators in the presence of electric fied, the degeneracy is lifted. This results in splitting of excitonic transisions cue to splitting in the exciton binding energy. For small spliting this will result in exciton broadening. Comparisons are presented for this broadening in symmetric and asymmetric guantum well structures as a function of electric field. The light hole exciton is found to broaden at an order of magnitude higher rate than the heavy hole exciton. Fielddependert experimental results for symmetric $\mathrm{GaAs} / \mathrm{Al}_{0.3} \mathrm{Ga}_{0.7} \mathrm{As}$ multiquantum well structures are carried out and support the theoretical results.
\end{abstract}

The study of exciton absorption peaks and their control through transverse electric field (quantum-confined Stark effeci) has recently become an important area of research. ${ }^{1-5}$ New phenomena and device concepts point to a bright future for exciton-based devices for optical processing. Modulator and photocurrent-controlled all-optical switches with significant contrast ratios have been demonstrated using III-V heterostructure technology. ${ }^{6-8}$

A key ingredient in the device performance of excitonicbased optical devices is exciton linewidth. Optical modulator contrast ratio as well as operation of the self-electro-optic effect device (SEED) ${ }^{7}$ depends strongly on this linewidth. Extrinsic effects such as interface roughness and well-to-well size fuctuation clearly contribute to the inhomogeneous line broadening ${ }^{9}$ while the phonons ${ }^{10}$ contribute to the homogeneous linewidth. However, another intrinsic source of tine broadening in optical modulators arises from the lifting of spin degeneracy of the hole states in quantum wells (Kramer's degeneracy) ${ }^{1-13}$ This occurs whenever the well is asymmetric and shows in the Kohn-Luttinger formalism ${ }^{12-14}$ due to heavy hole-light hole mixing term. The well asymmetry may be caused either by fabrication or by electric field.

To study the significance of this effect on exciton linewidths, we have solved the exciton problem in symmetric and asymmetric quantum wells in the presence of a transverse electric field. Since it was necessary to include the heavy hole (HH)-light hole (LH) coupling to study this phenomenon, we used the Kohn-Luttinger formalism 3,14 and applied it to the GaAs/AlGaAs system. Experimental studies were also carried out to study the exciton absorption in the presence of an electric field at low temperatures. The experimental and theoretical results are in reasonable agreement.

The subband positions for the electron and bole states are determined by solving the one-dimensional Schrödinger equation:

$$
-\frac{\hbar^{2}}{2 m} \nabla^{2} \Psi(k, z)+V(z) \Psi(k, z)=E \Psi(k, z),
$$

where $V(z)$ represents the potential barrier seen by the electrons.

To calculate the exciton binding energy and absorption constant, it is important to include the interaction between the light hole and heavy hole states, especially for the system we are considering here, since its states are very close. This is done via the Kohn-Luttinger Hamiltonian. ${ }^{14}$

Once the subband levels are known, the exciton problem is solved variationally and the absorption constant is determined by ${ }^{15}$

$$
\begin{aligned}
\alpha_{n m}= & \frac{4 \pi^{2} e^{2} n}{\eta m_{0} c W} \frac{1}{\hbar \omega}\left|\sum_{k_{\|}} G_{n n}\left(k_{\|}\right) \hat{\epsilon} \cdot p_{n m}\left(k_{\|}\right)\right|^{2} \\
& \times \delta\left(\hbar \omega-E_{m m}\right),
\end{aligned}
$$

where $\eta$ is the refractive index of the semiconductor, $\omega$ is the photon frequency, $\hat{\epsilon}$ is the polarization vector of the radiation, and $m, n$ are the hole and electron subband indices. The $p_{n m}\left(k_{\|}\right)$are optical matrix elements and the $G_{n m}\left(k_{\|}\right)$ are the Fourier components of the exciton envelope function and are determined by the solution of the exciton problem. The Dirac $\delta$ function is replaced by the broadening function to simulate the exciton linewidth.

$\mathrm{GaAs} / \mathrm{Al}_{0.3} \mathrm{Ga}_{0.7} \mathrm{As}$ multiquantum well sumples were grown in a Varian's Gen II MBE system. The well width was $100 \AA$ for the data reported here. $p-i(\mathrm{MQW})-n$ diodes were fabricated from these samples and the field-dependent absorption measurements were made after removing the substrate. The intrinsic region of the device was $2 \mu \mathrm{m}$ thick so that $1 \mathrm{~V}$ bias corresponded to a field of approximately $5 \mathrm{kV} /$ $\mathrm{cm}$.

Figure 1 shows the hole dispersion relation for a symmetric $100 \AA \mathrm{GaAs} / \mathrm{Al}_{0.3}, \mathrm{Ga}_{0.7}$ As quantum well structure at fields of $O$ and $70 \mathrm{kV} / \mathrm{cm}$. Note that for the symmetric structure, the hole subband states are doubly degenerate at 


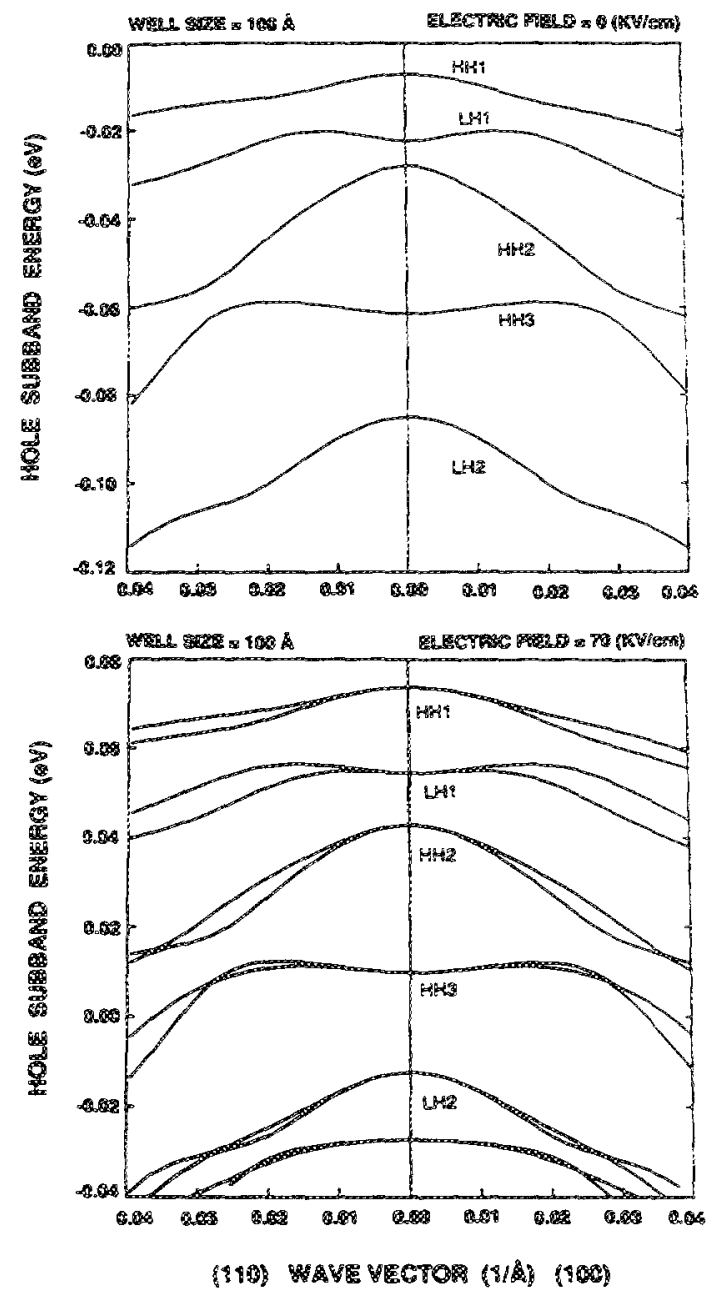

FIG. 1. Hole dispersion relations for a symmetric $100 \AA$ GaAs/ $\mathrm{Al}_{0.3} \mathrm{Ga}_{63}$ ? As quantum well structure at 0 and $70 \mathrm{kV} / \mathrm{cm}$.

zero field. In the presence of the electric field, the well is asymmetric and a splitting can be seen in the otherwise doubly degenerate $\mathrm{HH}$ and $\mathrm{LH}$ subband states. This splitting causes the exciton binding energy to be different for the two excitonic states. In the case of an asymmetric well, the structure is asymmetric even at zero bias and therefore exhibits splitting. In the presence of an electric field, the hole envelope functions may become either more asymmetric or less asymmetric depending upon the field direction. Figure 2 shows the calculated splitting for the symmetric 100 A quantum well as a function of field. We note that the broadening of the LHI exciton is much greater than the $\mathrm{HH}$ exciton because of the peculiar $\mathrm{LH} 1$ dispersion relation (as can be seen in Fig. 1). The LH1 states have negative mass due to the strong hole mixing effects and the $\mathrm{LH}$ l exciton mass is consequently very large. Thus a small change in the light hole mass causes a large change in the exciton effective mass and consequently in the $\mathrm{LH}$ binding energy.

From the experimentally observed field-dependent $a b$ sorption inset of Fig. 2, we have extracted the $\mathrm{HH}$ and $\mathrm{LH}$ ? exciton linewidth at $77 \mathrm{~K}$ where we expect that at zero bias the linewidth will be dominated by inhomogeneous broadening due to interface roughness and well-to-well size fuctuations. To determine the effect of Kramer's degeneracy lif-

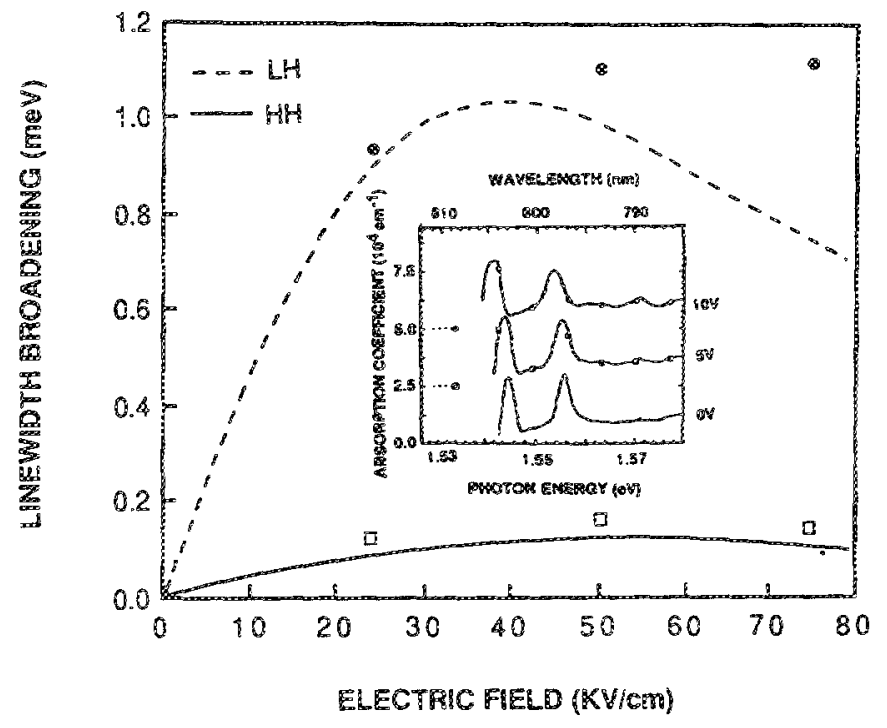

FEG. 2. Linewidth ( = spliting in exciton energies) calculated for $100 \mathrm{~A}$ GaAs $/ \mathrm{Al}_{03} \mathrm{G}_{40}$, As quantum well as a function of electric field. Also shown are experimentally observed absorption spectra and linewidths extracted from the data using Exss. (3)-(5).

ing on the exciton broadening, we removed the effects of inhomogeneous broadening from the total linewidth. For this we have calculated:

$$
\Delta_{\mathrm{int}}^{\mathrm{HH}}(E)=\Delta^{\mathrm{HH}}(E)-B^{\mathrm{HH}}(\bar{F}) \Delta^{\mathrm{HH}}(0),
$$

and

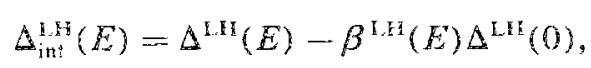

where $\Delta^{\text {ikt }}(E)$ and $\Delta^{L i t}(E)$ are the field-dependent linewidths for the $H H 1$ and $L H 1$ transitions, and $\beta^{\mathrm{HHI}}(E)$ and $\beta^{\mathrm{L} . H}(E)$ represent the effect of electric field on inhomogeneous broadening. Note that $\beta^{\mathrm{HH}}(E)$ and $\beta^{\mathrm{LH}}(E)$ both increase with the field ${ }^{9}$ and that $\beta^{\mathrm{LH}}(E)$ is less than $\beta^{\text {iHI }}(E)$. In generals

$$
\left.\beta \cong \frac{\partial E_{\mathrm{cx}}(E)}{\partial w}\right|_{w-w_{0}} /\left.\frac{\partial E_{\mathrm{e}}\left(E^{*}=0\right)}{\partial w}\right|_{w-u_{w}},
$$

where $E_{\mathrm{ex}}$ is the exciton energy and $w_{0}$ is the well size.

Thus the quantities in Eqs. (3) and (4) represent the intrinsic field dependent broadening. Since elcctron and hole iunneling rates are quite low at these fields, these values essentially represent the broadening due to Kramer's degeneracy. We have plotted these points in Fig. 2 for the HHI and L 1 l excitons. We note that in agreement with theoretical predictions, the broadening is much stronger than for the LH1 excitonic state than for the HHI state. We also note that it is difficult to extract linewidth from absorption data, and so we expect our values to have $\sim \pm 15 \%$ error. Howcver, since the relative broadening of $L H 1$ and $H H Y$ states is expected to remain fairly accurate, we find a fair agreement with our theoretical preaictions.

Finally, in Fig. 3 we have plotted the linewidth due to the ifting of $\mathrm{K}$ ramer's degeneracy for an asymmetric well which has the composition $\mathrm{Al}_{0.2} \mathrm{Ga}_{0.8} \mathrm{As} / \mathrm{GaAs}(100 \AA \mathrm{A}) /$ $\mathrm{Al}_{0.4} \mathrm{Ga}_{0.6} \mathrm{As}$. In this case, the well is asymmetric at zero bias and, as shown in Fig. 3 , has a finite splitting at zero bias. The splitting is also found to be dependent on the direction of 


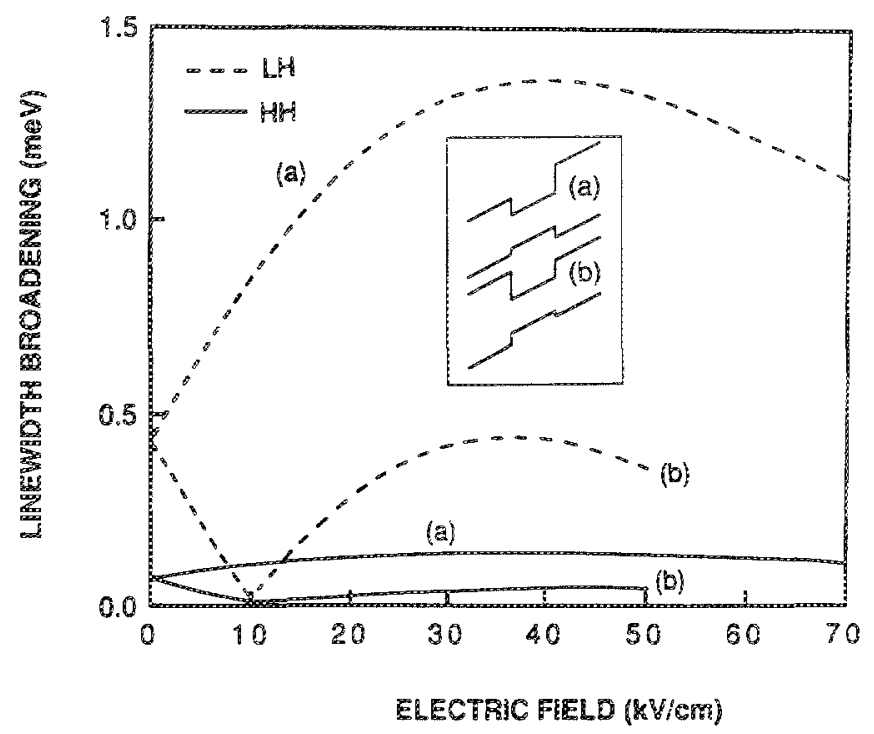

FIG. 3. Linewidth colculated for asymmetric quantum well (shown in insot) for two difierent directions of the transverse electric field.

the electric field as expected. When the electric field is in the direction which initially makes the hole wave function more symmetric with bias, the splitting goes through a minimum as shown. In this case we show results for fields only up to 50 $\mathrm{kV} / \mathrm{cm}$ since the exciton is dissociated at higher fields.

In summary, we have discussed theoretical and experimental consequences of $\mathrm{K}$ ramer's degeneracy in optical modulators. Due to strong hole mixing, the effect on the 11 state is much more dominant than on the HHI state. Asym- metric wells can be designed so that this broadening is minimum at a specific electric field.

The work at the University of Nichigan was supported by the NSF Lightwave Technology Program under grant ECE 8610803. The Rockwell work was carried out under U.S. Army's SDC contract No. DASG60-86-C-0095. We gratefuly acknowledge the help of Dr. David L. Miller and Gerald J. Sullivan.

'i. H. Wood, C. A. Burrus, D. A. B. Milkr, D. S. Chemla, T. C. Damen. A. C. Gossard, and W. Wegman, Appl. Phys. Leit. 44, 16 (1984).

${ }^{2}$ P. Li Kam Wa, J. I. Sitch, N. J. Masons, J. S. Roberts, and P. N. Robson, Electron. Lett. 49, 135 (1986).

${ }^{3}$ T. H. Wood, C. A. Burrus, R. S. Tuker, J. S. Weiner, D. A. B. Miller, D. S. Chemla, T. C. Damen, A. C. Gosard, and W. Wiegman, Electron. Let. 21. 093 (1985).

TN. Peyghambarian and H. M. Gibbs, Opt. lng. 24, 68 (1985)

${ }^{5}$ T. E. Van Eck, P. Chu, W. S. C. Chang, and H. I. Wieder, Appl. Phys. L.ett. 49. $\$ 35$ (1986).

${ }^{6}$ D. A. B. Miner, D. S. Chemla, T. C. Damer, T. H. Wood, C. A. Burrus, A. C. Gossard, and W. Witgmann, MEEE J. Quantum Eleatron. QE-âl, $1462(1985)$

7D. A. W. Miller, D. S. Chemia, T. C. Damen, A. C. Gossard, W. Wiegmann, T. H. Wood, and C. A. Burrus, Appl. Phys. Lett. 45, 13 (1984).

${ }^{8}$ P. Whactley, M. Whitehead, J. E. Midwinter, B. Mistry, M. A. Pate, and J. S. Robetts, Opt. Lett. 12,784 (1987).

'S. Hong and J. Singh, Appl. Phys. Lett. 49, 331 (1986).

10J. Lee, E. S. Koteles, and M. O. Vassell, Bhys. Rev. B 33, 5512 (1986).

'Z. Schlesinger, S. J. Allen, Y. Yafet, A. C. Gossard, and W. Wiegmann, Phys. Rev. 1332, 5231 (1985).

${ }^{12}$ D. A. Broido and L. J. Shan, Phys. Rev. B 31,888 (1985).

${ }^{13}$ S. Hong, M. Jatie, and J. Singh, IEEE J. Quantum Elew ron. QE-23, 2181 (1987).

${ }^{14} 3$. M. Luttinger and W. Kohn, Phys. Rev. 97, 869 (1955)

"P. Lawaerz, Phys. Rev. B A, 3460 (1971). 